\title{
Pentaquarks: Status and Perspectives for Lattice Calculations
}

\author{
Shoichi Sasaki ${ }^{\mathrm{a}}$ \\ ${ }^{a}$ Department of Physics, University of Tokyo, Tokyo 113-0033, Japan
}

The present status of pentaquark spectroscopy in lattice QCD is reviewed. This talk also includes a brief introduction of pentaquark baryons.

\section{Introduction}

The quantum chromo-dynamics (QCD) may not preclude the presence of the multi-quark hadrons such as tetraquark $(q q \bar{q} \bar{q})$, pentaquark $(q q q q \bar{q})$, dibaryon $(q q q q q q)$ and so on, because of the color confinement. Especially, we are let to be interested in exotic multi-quark hadrons, which should have exotic quantum numbers. Now, let us address ourself to the pentaquark state. Consider the $S U(3)$ flavor case. Pentaquark states should form six different multiplets:

$$
\begin{aligned}
3_{f} \otimes 3_{f} & \otimes 3_{f} \otimes 3_{f} \otimes \overline{3}_{f} \\
& =1_{3} \oplus 8_{8} \oplus 10_{4} \oplus \overline{10}_{2} \oplus 27_{3} \oplus 35_{1}
\end{aligned}
$$

where subscripts in the right hand side denote the number of degeneracy in each multiplet. The first three multiplets are common in the case of usual baryons. However, the last three multiplets; antidecuplet, 27-plet and 35-plet, are distinct irreducible representations since those multiplets have an apparent exotic quantum-number such as strangeness +1 . Needless to say, $S=+1$ baryon can not be accommodated by usual baryons. Other possible exotic quantum-numbers are represented as stars in Fig. 11 Of course, one cannot predict which multiplet is preferred for the possible $S=+1$ pentaquark baryon, within the group theoretical argument. The Skyrme model [1] and the chiral soliton model 2], however, predict that the lowest $S=+1$ state appears uniquely in the antidecuplet and its spin and parity is spin-half and positive parity.

Recently, the LEPS collaboration at Spring-8 has observed a very sharp peak resonance in the $K^{-}$missing-mass spectrum of the $\gamma n \rightarrow n K^{+} K^{-}$ reaction on ${ }^{12} \mathrm{C}$ [3. The observed resonance should have strangeness +1 . Thus, $\Theta^{+}(1540)$ cannot be a three quark state and should be an exotic baryon state with the minimal quark content $u$ udd $\bar{s}$. The peak position is located at $1540 \mathrm{MeV}$ with a very narrow width. Those are quite consistent with the chiral-soliton model's prediction [2]. This discovery is subsequently confirmed by other experiments 45$]^{1}$. Experimentally, spin, parity and isospin are not determined yet. Non-existence of a narrow resonance in $p K^{+}$ channel indicates that possibility of $I=1$ has been already ruled out 35.

Many theoretical studies of pentaquarks are also triggered by the discovery of the $\Theta^{+}(1540)$. I introduce the most reputed model proposed by Jaffe and Wilczek 77. In the naive quark models, the low-lying pentaquark state should have spin-1/2 and negative parity. However, in this case, the pentaquark baryon just falls apart into $K N$ in a $\mathrm{S}$-wave. It is difficult to explain its very narrow width. Jaffe and Wilczek propose a simple idea to flip the parity of the low-lying pentaquark. Suppose there is the strong diquark correlation. The spin- 0 , color triplet and flavor triplet diquark would be favored within the simple one gluon exchange. The pentaquark can be composed of two identical bosons (diquarks) and one antiquark. However, the anti-symmetrization in terms of color, requires relative odd number's angular-momentum between the pairs of identical bosons. Otherwise, the wave function of the pentaquark state should be vanished. Resulting parity of the low-lying pentaquark is same as the

\footnotetext{
${ }^{1}$ It should be noted, however, that the experimental evidence for the $\Theta^{+}(1540)$ is not very solid yet since there are a similar number of negative results to be reported [6].
} 


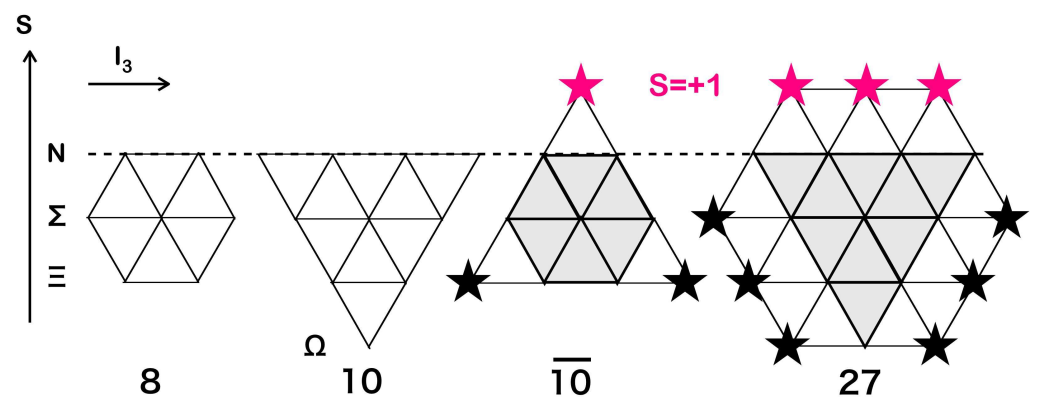

Figure 1. Weight diagrams for possible pentaquark multiplets. Stars represent the states which have exotic quantum numbers (electric charge and strangeness).

chiral soliton model [7. In addition, the $S=+1$ baryon is uniquely assigned to antidecuplet in this description.

Consequently, correlated quark models, i.e. the diquark model, may accommodate the positiveparity isosinglet pentaquark $\Theta(u u d d \bar{s})$ as same as the chiral soliton model. However, there are essential differences between the diquark model and the chiral soliton model. In correlated quark models, one cannot fully discriminate the other multiplet such as octet. If apart from the $S U(3)$ flavor limit, the mixing between octet and antidecuplet should be taken into account. Jaffe and Wilczek advocate the ideal mixing case, which is favored in the vector meson spectrum [7]. The ideal mixing provides the different prediction for the mass of possible exotic $\Xi_{3 / 2}^{--}$state, which should have isospin-3/2. Furthermore, the Roper resonance, $N(1440)$, can be accommodated in the diquark model owing to the ideal mixing [7].

A candidate of the isospin- $3 / 2$ pentaquark state has been reported by the NA49 collaboration 8 . They observed the sharp peak resonance, which has exotic quantum numbers, in $\Xi \pi$ missing mass analysis. The observed mass is located somewhat between predictions made by the chiral soliton model and the diquark model. In this experiment, the isospin partner, $\Xi_{3 / 2}^{0}$ is also observed. It should be pointed out that this discovery is not confirmed yet by other experiments [6].

I shortly mention about charm or bottom ana$\log$ of the $\Theta$ state. If anti-strange quark is re- placed by a heavier antiquark, what is going to be happened. Several models predict that charm or bottom analog of the pentaquark is expected to be a bound state [79]. Recently, the discovery of the exotic anti-charmed baryon $(u u d d \bar{c})$ has been announced by the H1 collaboration [10]. However, the observed state is higher than the $D N$ threshold and also the $D^{*} N$ threshold against several model predictions 79. Again, this discovery has not been confirmed yet by other experiments.

\section{Lattice pentaquark spectroscopy}

If the pentaquark baryons really exist, such states must emerge directly from first principles, QCD. Of course, what we should do is to confirm the presence of the pentaquarks by lattice QCD. Experimentally, it is rather difficult to determine the parity of the $\Theta^{+}(1540)$. Thus, lattice QCD has a chance to answer the undetermined quantum numbers before experimental efforts. Lattice QCD has also a feasibility to predict the masses for undiscovered pentaquark baryons. I stress that there is substantial progress in lattice study of excited baryons recently [11. Especially, the negative parity nucleon $N^{*}(1535)$, which lies close to the $\Theta^{+}(1540)$, has become an established state in quenched lattice QCD [1112]. Here I report that quenched lattice QCD is capable of studying the $\Theta^{+}(1540)$ as well.

Indeed, it is not so easy to deal with the $q q q q \bar{q}$ state rather than usual baryons $(q q q)$ and 
mesons $(q \bar{q})$ in lattice QCD. The $q q q q \bar{q}$ state can be decomposed into a pair of color singlet states as $q q q$ and $q \bar{q}$, in other words, can decay into two hadron states even in the quenched approximation. For instance, one can start a study with a simple minded local operator for the $\Theta^{+}(1540)$, which is constructed from the product of a neutron operator and a $K^{+}$operator such as $\mathcal{O}=\varepsilon_{a b c}\left(d_{a}^{T} C \gamma_{5} u_{b}\right) d_{c}\left(\bar{s}_{e} \gamma_{5} u_{e}\right)$. The two-point correlation function composed of this operator, in general, couples not only to the $\Theta$ state (single hadron) but also to the two hadron states such as an interacting $K N$ system [1314. Even worse, when the mass of the $q q q q \bar{q}$ state is higher than the threshold of the hadronic two-body system, the two-point function should be dominated by the two hadron states. Thus, a specific operator with as little overlap with the hadronic twobody states as possible is desired in order to identify the signal of the pentaquark state in lattice QCD. Once one can identify the pentaquark signal in lattice QCD, to determine the parity of the $\Theta^{+}(1540)$ is the most challenging issue at present. Thus, it is necessary to project out the parity eigenstate from given lattice data precisely. In the following subsections, I discuss three related issues

\subsection{Estimation of the $K N$ threshold}

The experimentally observed $\Theta^{+}(1540)$ state is clearly a resonance state. However, its mass is near the $K N$ threshold. We could manage to calculate the pentaquark as a bound state if its parity were positive.

I recall that all momenta are quantized as $\vec{p} L=2 \pi \vec{l}\left(\vec{l} \in \mathbf{Z}^{3}\right)$ on lattice in finite volume (the spacial extent $L$ ) with the periodic boundary condition (PBC).$^{2}$ Thus, the spectrum of energies of two hadron states such as $K N$ states with zero total momentum should be discrete and these energies are approximately equal to values, which are evaluated in the noninteracting case:

$E_{n}^{K N}=\sqrt{M_{N}^{2}+p_{n}^{2}}+\sqrt{M_{K}^{2}+p_{n}^{2}}$

${ }^{2}$ Of course, this quantization condition may change to $\vec{p} L+2 \delta(p)=2 \pi \vec{l}$ with a scattering phase shift $\delta(p)$ by an interaction between two hadrons. However, the $K N$ channel has very weak interactions as is well known experimentally. One may omit this shift for a crude estimation.

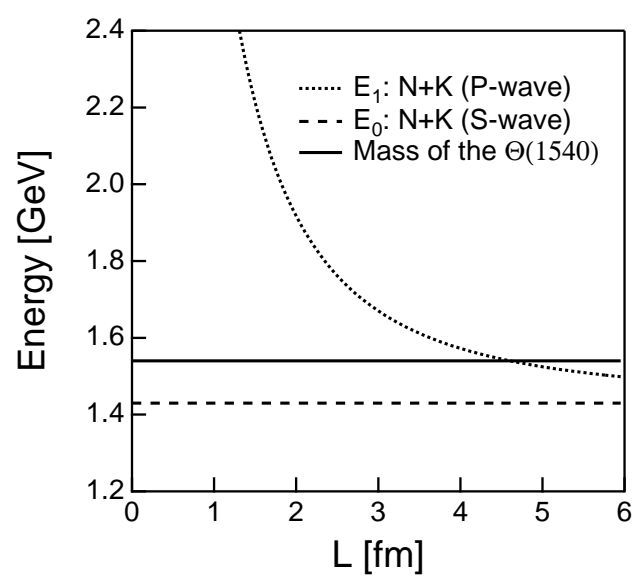

Figure 2. The $\mathrm{S}$-wave and $\mathrm{P}$-wave $K N$ threshold energies on a lattice of spatial extent $L$. In the case of $L \leq 4.6 \mathrm{fm}$, the mass of the $\Theta^{+}(1540)$ is lower than the $\mathrm{P}$-wave $K N$ threshold.

where $p_{n}=\sqrt{n} \cdot 2 \pi / L$ and $n \in \mathbf{Z}$. The positive parity $\Theta$ state decay into $K N$ in a $\mathrm{P}$-wave where the $K N$ system should have a nonzero relative momentum. The $\mathrm{P}$-wave $K N$ threshold is simply estimated at an energy level $E_{1}$, which is evaluated with the smallest nonzero momentum $p_{1}=2 \pi / L$ in Eq. (1). This energy level $E_{1}$ can be lifted by decreasing spatial extent as depicted in Fig. 2 while the lowest energy level $E_{0}$, which corresponds to the S-wave $K N$ threshold, remains unchanged. The level crossing between $E_{1}$ and the $\Theta$ mass takes place around $4.6 \mathrm{fm}$ in this crude estimation. It implies that the positive parity $\Theta$ state may become a bound state in the typical lattice size of currently available lattice calculations, i.e. $L \approx 2-3 \mathrm{fm}$.

\subsection{Choice of operators}

For the case of the negative parity $\Theta$ state, the presence of the $K N$ scattering state must complicates the study of pentaquarks in lattice QCD. One should choose an optimal operator, which couples weakly to the $K N$ scattering state, in order to access the pentaquark state above the (S-wave) $K N$ threshold.

For this direction, I would like to recall that 


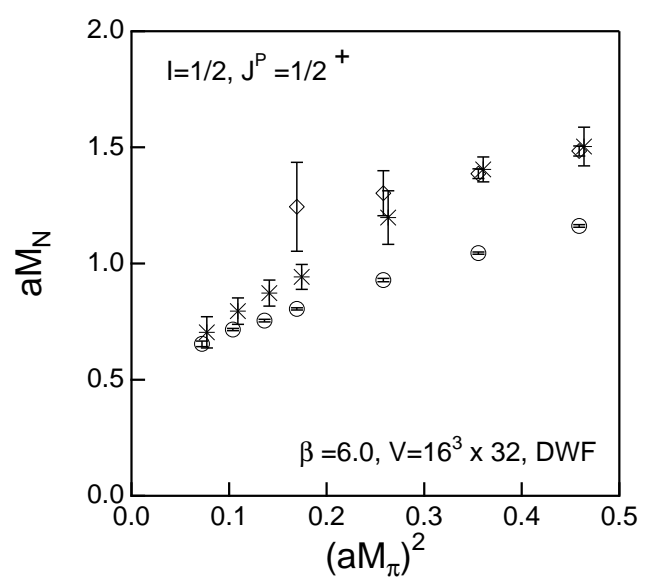

Figure 3. Comparison of the fitted mass from $\left\langle\mathcal{O}_{1}^{N} \overline{\mathcal{O}}_{1}^{N}\right\rangle(\circ),\left\langle\mathcal{O}_{2}^{N} \overline{\mathcal{O}}_{2}^{N}\right\rangle(\diamond)$ and the cross correlation $\left\langle\mathcal{O}_{1}^{N} \overline{\mathcal{O}}_{2}^{N}+\mathcal{O}_{2}^{N} \overline{\mathcal{O}}_{1}^{N}\right\rangle(*)[12$.

the less known observation in the spectroscopy of the nucleon. There are two possible interpolating operators for the $I=1 / 2$ and $J^{P}=$ $1 / 2^{+}$state; $\mathcal{O}_{1}^{N}=\varepsilon_{a b c}\left[u_{a}^{T} C \gamma_{5} d_{b}\right] u_{c}$ and $\mathcal{O}_{2}^{N}=$ $\varepsilon_{a b c}\left[u_{a}^{T} C d_{b}\right] \gamma_{5} u_{c}$, even if one restricts operators to contain no derivatives and to belong to the $\left(\frac{1}{2}, 0\right) \oplus\left(0, \frac{1}{2}\right)$ chiral multiplet under $S U(2)_{L} \otimes$ $S U(2)_{R}[12]$. Of course, two operators have the same quantum number of the nucleon. The first operator $\mathcal{O}_{1}^{N}$ is utilized conventionally in lattice QCD since the second operator $\mathcal{O}_{2}^{N}$ vanishes in the non-relativistic limit. It implies that the second operator is expected to have small overlap with the nucleon $\left|\left\langle 0\left|\mathcal{O}_{2}^{N}\right| \mathrm{Nucl}\right\rangle\right| \approx 0$. Indeed, the mass extracted from the correlator constructed by the second operator $\mathcal{O}_{2}^{N}$ exhibit the different mass from the nucleon mass [1215] as shown in Fig. 3] The operator dependence on a overlap with desired state is evident, at least, in the heavy quark regime, while the cross correlation suggests that the small overlap with the nucleon might be no longer robust in the light quark regime where is far from the non-relativistic description [12].

\subsection{Parity projection}

The intrinsic parity of the local baryon operator can be defined by the parity transformation of internal quark fields as

$\mathcal{P} \mathcal{O}^{(\eta)}(\vec{x}, t) \mathcal{P}^{\dagger}=\eta \gamma_{4} \mathcal{O}^{(\eta)}(-\vec{x}, t)$,

where $\eta= \pm 1$. However, due to the relation $\mathcal{O}^{(+)}=\gamma_{5} \mathcal{O}^{(-)}$for the local baryon operator, the resulting two-point correlation functions are also related with each other as $\left\langle\mathcal{O}^{(+)}(x) \overline{\mathcal{O}}^{(+)}(0)\right\rangle=$ $-\gamma_{5}\left\langle\mathcal{O}^{(-)}(x) \overline{\mathcal{O}}^{(-)}(0)\right\rangle \gamma_{5}$. This means that the two-point correlation function composed of the local baryon operator can couple to both positiveand negative-parity states. However, I note that anti-particle contributions of opposite parity states is propagating forward in time. Thus, the $+/-$ parity eigenstate in the forward propagating contributions is obtained by choosing the appropriate parity projection $\left(1 \pm \eta \gamma_{4}\right) / 2$, which is given in reference to the intrinsic parity of operators, $\eta$. Details of the parity projection are described in Ref. [12.

\section{First exploratory studies}

\subsection{Local pentaquark operators}

As I remarked previously, an optimal operator, which couples weakly to the $K N$ scattering state, would be required to explore the pentaquark baryons in lattice QCD. For this purpose, two types of local pentaquark operator for isosinglet state are proposed in the first two studies. One is a color variant of the simple product of nucleon and kaon operators,

$\mathcal{O}_{I=0}^{(-)}=\varepsilon_{a b c}\left[u_{a}^{T} C \gamma_{5} d_{b}\right]\left\{u_{e}\left(\bar{s}_{e} \gamma_{5} d_{c}\right)-(u \leftrightarrow d)\right\},(3)$

which is proposed by Csikor et al. 16

The other is proposed by Sasaki [17] as in a rather exotic description guided by the diquarkdiquark-antiquark structure:

$\mathcal{O}_{I=0}^{(\eta)}=\varepsilon_{a b c} \varepsilon_{a e f} \varepsilon_{b g h}\left[u_{e}^{T} C \Gamma_{1} d_{f}\right]\left[u_{g}^{T} C \Gamma_{2} d_{h}\right] C \bar{s}_{c}^{T}(4)$

where $\Gamma_{1,2}=1, \gamma_{5}, \gamma_{5} \gamma_{\mu}$ (but $\Gamma_{1} \neq \Gamma_{2}$ ) and the superscript " $\eta$ " stands for the intrinsic parity of the operator. There are three kinds of diquarkdiquark-antiquark operator in this description, which are useful for the extended study with the $3 \times 3$ correlation matrix analysis. More details of construction of the diquark-diquark-antiquark operator are described in Ref. [17. 


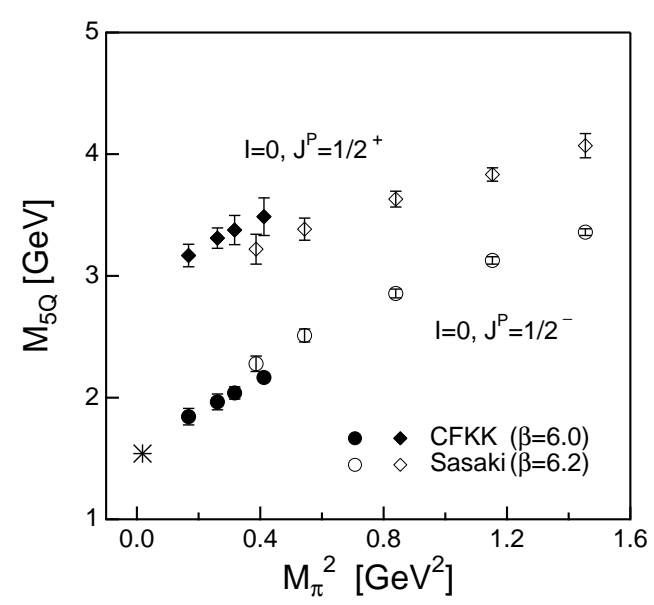

Figure 4. Masses of the isosinglet $S=+1$ baryons with both positive- and negative-parity as functions of pion mass squared 1617. The experimental value for $\Theta^{+}(1540)$ is marked with a star.

In an exploratory study, one may assume that those interpolating operators have smaller overlap with the $K N$-scattering state than the simple product of nucleon and kaon operators, at least, in the heavy quark regime. Because, in the nonrelativistic limit, all of them give rise to the different wave function from the $K N$ two-hadron system.

\subsection{Results}

The first two lattice studies were performed with the Wilson gauge action and the Wilson fermion action at the almost same box size $L \simeq$ 2.0-2.2 fm. The lattice spacing Csikor et al. use is rather coarse than that of Sasaki, but their calculation was employed with relatively lighter pion masses $\left(M_{\pi} \sim 0.4-0.6 \mathrm{GeV}\right)$. The main difference between two studies is the choice of pentaquark operators.

After some initial confusion about the parity assignment $^{3}$, both calculations agreed that the lowest state of the isosinglet $S=+1$ baryons has the negative parity as shown in Fig. 4 The main results from the first two exploratory studies can

$\overline{{ }^{3} \text { See a footnote in Ref. }}$ [6]. be summarized as follows.

- The current lattice simulations seem to give no indication of a pentaquark in the positive parity channel to be identified with the $\Theta^{+}(1540)$.

- The negative parity channel can easily accommodate a pentaquark with a mass close to the experimental value.

Therefore, both authors conclude that the exploratory lattice study favors spin-parity $(1 / 2)^{-}$ and isospin 0 for the $\Theta^{+}(1540)$. In Ref. [17, the anti-charmed analog of the $\Theta$ state was also explored. It is found that the $\Theta_{c}(u u d d \bar{c})$ lies much higher than the $D N$ threshold, in contrast to several model predictions 79]. More detailed lattice study would be desirable to clarify the significance of those observations.

\section{Subsequent lattice studies}

There are four subsequent lattice studies of pentaquark spectroscopy to be found in the literature 18192021 . Other two preliminary results had been also reported at some conferences 2223 . I give a short review of those results as follows.

Recently, Kentucky group performed their simulations near the physical pion mass region with overlap fermions 18. However, they choose the simple minded operator as the product of nucleon and kaon operators to explore the pentaquark baryons. Instead, the sequential constrained fitting method is applied in their analysis to disentangle the pentaquark signal from towers of $K N$ scattering state. They also check carefully the volume dependence of the spectral weight. They claim as follows: Only towers of $K N$ scattering state are seen in the negative parity channel. They confirmed that ground state in either parity channels has a characteristic volume dependence on the spectral weight, which should have $1 / L^{3}$ dependence for two particles. They also confirm that the ghost contribution from $K N \eta^{\prime}$ state appears from pion mass less than around $0.3 \mathrm{GeV}$ in the positive parity channel. Their final conclusion is that there is no sign of pentaquark signal in either parity channels in their 
Table 1

Summary of the present status of lattice pentaquark spectroscopy

\begin{tabular}{lccc}
\hline author(s) and reference & signal & parity of pentaquark & kind of operator \\
\hline \hline Csikor et al. [16] & Yes & negative & color variant of $K N$ \\
Sasaki [17] & Yes & negative & diquark-diquark-antiquark \\
Mathur et al. [18] & No & N/A & simple $K N$ \\
Chiu-Hsieh [19] & Yes & positive & diquark-diquark-antiquark \\
Ishii et al. [20] & No & N/A & diquark-diquark-antiquark \\
Alexandrou et al. [21] & Yes & negative & diquark-diquark-antiquark \\
MIT group [22] & Yes & negative & diquark-diquark-antiquark \\
YITP group [23] & Yes & negative & simple $K N$ and color variant of $K N$ \\
\hline
\end{tabular}

calculation. However, it seems that their results are consistent with the experimental fact that the $\Theta^{+}(1540)$ state has not yet been found in the $K N$ scattering data as an elastic resonance [24].

Another negative results against the first two studies are reported by TIT group 20 . First, they trace calculations of Ref. [17] on an anisotropic lattice with the $O(a)$ improved Wilson fermion action. They confirm that the lowest energy state appears in the negative parity channel. In their study, a new method is proposed to lift up the S-wave $K N$ threshold by imposing a hybrid boundary condition (HBC) in the spatial direction and it is also applied in their calculation. They found that the plateau of the lowest energy state is shifted in the effective mass plot by changing the spatial boundary condition from $\mathrm{PBC}$ to $\mathrm{HBC}$ as is expected in the case of the two hadron states. However, their calculations are employed with smeared sources, which are apparently optimized for the ground state of usual hadrons, i.e. nucleon and kaon. This optimization may enhance the signal of the $K N$ scattering states rather than that of the pentaquark state. Indeed, the resulting effective mass plots for the pentaquark do not show any effectiveness of their smearing in the earlier Euclidean time region, in contrast to the case of usual baryons. However, of course, their method, i.e. the hybrid boundary method, is quite promising to distinguish between the pentaquark state and the $K N$ scattering state.

There are several positive results for the first two studies. Cyprus group performed careful studies concerning the volume dependence of the spectral weight and then found that their observed state seems to be a single hadron state, i.e. the pentaquark state 21. MIT group formulates eight possible local operators based on the diquark-diquark-antiquark structure to perform the $4 \times 4$ correlation matrix analysis in both isosinglet and isotriplet channels as a extension of Sasaki's proposal 22. Their preliminary results are currently obtained from double exponential fits on data for each single operator. They confirmed results of the first two studies at their exploratory stage. YITP group has started the $2 \times 2$ correlation matrix analysis with the simple minded operator as the product of nucleon and kaon operators and its color variant [23]. Their preliminary results support that there is an indication of the presence of the pentaquark state near the lowest $K N$ scattering state in the negative parity channel.

Finally, I comment on Chiu-Hsieh's results. In the first version of Ref. 19], they initially misunderstood the parity assignment. Then, their results are completely opposite to ones of any other studies where the lowest energy state appears in the negative parity channel. In the second version, they corrected this apparent error and reanalyzed their data. However, their final conclusion remains unchanged. They insist that the spinparity of the $\Theta^{+}(1540)$ state is most likely $(1 / 2)^{+}$. This conclusion seems to attribute to their crude chiral extrapolation. 


\section{Summary and Outlook}

Table 1 represents a summary of the present status for each lattice calculation. The first conclusion of the first two studies as summarized in Sec. 3.2 is confirmed by subsequent lattice studies. The currently important issue is whether or not to establish the presence of the $\Theta^{+}(1540)$ in the negative parity channel. It is necessary for this to disentangle the pentaquark signal from the $K N$ scattering states completely . The correlation matrix analysis is strongly required to separate the $K N$ scattering state and isolate the pentaquark state. We also should check the volume dependence of the spectral weight. Probably, the hybrid boundary condition is helpful to identify the pentaquark state as a single hadron state. Needless to say, we ought to try non-local types of pentaquark operator in order to verify whether there is no indication of the $\Theta^{+}(1540)$ in the positive parity channel.

Finally I stress that all present results should be regarded as exploratory. Indeed, much detail studies are in progress in each group. Thus, I had better to conclude that the following questions still remain open:

- Does the spectrum of QCD possess the $\Theta^{+}(1540)$ ?

- What is spin and parity of the $\Theta^{+}(1540)$ ?

- Are there other pentaquark baryons, e.g. the charm (bottom) pentaquark or the spinorbit partner of the $\Theta^{+}(1540)$ ?

There are many exiting issues to be explored.

\section{Acknowledgments}

This talk has benefited from conversation and correspondence with T.-W. Chiu, Z. Fodor, S. Katz, F.-X. Lee, K.-F. Liu, N. Mathur and J. Negele. The author is supported by JSPS Grantin-Aid for Encouragement of Young Scientists (No. 15740137).

\section{REFERENCES}

1. A. V. Manohar, Nucl. Phys. B 248, 19 (1984); M. Chemtob, Nucl. Phys. B 256, 600 (1985).
2. D. Diakonov, V. Petrov and M. V. Polyakov, Z. Phys. A 359, 305 (1997).

3. LEPS Collaboration, Phys. Rev. Lett. 91, 012002 (2003).

4. DIANA Collaboration, Phys. Atom. Nucl. 66, 1715 (2003); CLAS Collaboration, Phys. Rev. Lett. 91, 252001 (2003).

5. SAPHIR Collaboration, hep-ex/0307083

6. For a recent experimental review, see K. Hicks, hep-ph/0408001 references therein.

7. R. L. Jaffe and F. Wilczek, Phys. Rev. Lett. 91, 232003 (2003).

8. NA49 Collaboration, Phys. Rev. Lett. 92, 042003 (2004).

9. F. Stancu, Phys. Rev. D 58, 111501 (1998); M. Karliner and H. J. Lipkin, hep-ph/0307343

10. H1 Collaboration, Phys. Lett. B 588, 17 (2004).

11. For recent reviews, see S. Sasaki, Prog. Theor. Phys. Suppl. 151, 143 (2003), nucl-th/0305014 C. Morningstar, nucl-th/0308026 D. B. Leinweber et al., nucl-th/0406032 and references therein.

12. S. Sasaki, T. Blum and S. Ohta, Phys. Rev. D 65, 074503 (2002).

13. M. Lüscher, Commun. Math. Phys. 105, 153 (1986).

14. M. Fukugita et al., Phys. Rev. D 52, 3003 (1995).

15. K. C. Bowler et al., Nucl. Phys. B 240, 213 (1984); D. B. Leinweber, Phys. Rev. D 51, 6383 (1995).

16. F. Csikor, Z. Fodor, S. D. Katz and T. G. Kovacs, JHEP 0311, 070 (2003), hep-lat/0309090

17. S. Sasaki, Phys. Rev. Lett. 93, 152001 (2004), hep-lat/0310014

18. N. Mathur et al., hep-ph/0406196

19. T. W. Chiu and T. H. Hsieh, hep-ph/0403020

20. N. Ishii et al., hep-lat/0408030.

21. C. Alexandrou et. al., hep-lat/0409065

22. J. Negele, Talk presented at QNP2004.

23. T.T. Takahashi, Talk presented at Pentaquark2004.

24. R. A. Arndt et al., Phys. Rev. C 68, 042201 (2003) [Erratum-ibid. C 69, 019901 (2004)]. 